\title{
Smoking during Pregnancy Is a Predictor of Poor Perinatal Outcomes in Maternal Anorexia Nervosa: A Case Series and Single-Center Cross-Sectional Study in Japan
}

\author{
Kyoko Kasahara, ${ }^{1}$ Tetsuo Ono, ${ }^{1,2}$ Asuka Higuchi, ${ }^{1}$ Daisuke Katsura, ${ }^{1}$ \\ Kaori Hayashi, ${ }^{1}$ Shinsuke Tokoro, ${ }^{1}$ Shunichiro Tsuji, ${ }^{1}$ Fuminori Kimura ${ }^{1}$ and \\ Takashi Murakami ${ }^{1}$ \\ ${ }^{1}$ Department of Obstetrics and Gynecology, Shiga University of Medical Science, Otsu, Shiga, Japan \\ ${ }^{2}$ Department of Obstetrics and Gynecology, Omihachiman Community Medical Center, Omihachiman, Shiga, \\ Japan
}

\begin{abstract}
Eating disorders are common psychiatric disorders among women of reproductive age, and the prevalence of eating disorders has been increasing over time in Japan and other countries. The aim of the present study was to assess perinatal outcomes in maternal anorexia nervosa in Japan and to explore methods to improve perinatal outcomes. This study consists of a case series describing 13 single pregnancies of 11 women with a history of anorexia nervosa, and a cross-sectional study comparing 13 cases with 240 healthy controls. In the case group, nine cases conceived while underweight, including three who had fertility treatment. Anorexia symptoms during pregnancy were quite common, and pregnant smokers presented with extremely disturbed eating behaviors. In a cross-sectional study, premature birth and the standard deviations from the mean birth weight and mean head circumference at birth were evaluated as outcome measures. The adjusted odds ratios or the adjusted differences between two means for the above outcomes were estimated by two approaches: multivariate models and matching analysis. Statistical analysis showed that maternal anorexia nervosa was associated with an increased risk of premature birth and symmetric growth restriction mediated by low pre-pregnancy body mass index and poor gestational weight gain which were adjusted as confounders. Smoking during pregnancy was a potential indicator of abnormal eating behavior and could be predictive of poor perinatal outcomes. We therefore conclude that remission of anorexia nervosa before pregnancy could improve perinatal outcomes through both normal nutrition and smoking cessation. Fertility treatment while underweight is not recommended.
\end{abstract}

Keywords: anorexia nervosa; low birth weight infant; pregnancy; premature birth; smoking Tohoku J. Exp. Med., 2020 April, 250 (4), 191-200.

\section{Introduction}

The prevalence of eating disorders among Japanese women has been increasing over time (Nakai et al. 2014), similarly to other countries (Kimmel et al. 2016; O'Brien et al. 2017). Eating disorders are common psychiatric disorders among women of reproductive age, and a variety of pregnancy-related complications have been reported (Rondo et al. 2003; Koubaa et al. 2005; Micali et al. 2007, 2012, 2016; Bulik et al. 2009; Hoffman et al. 2011; Linna et al. 2014; Kimmel et al. 2016; O’Brien et al. 2017). However, to our knowledge, despite the increasing numbers of patients, no studies on maternal eating disorders in Japan have been reported in the English or Japanese literature. Because the incidence of eating disorders might be related to race, socioeconomic status, and cultural factors (O'Brien et al. 2017; Nakai et al. 2018), the clinical characteristics and perinatal outcomes of Japanese women with a history of eating disorders need to be clarified in order to decrease the risk of poor health in mothers and children.

Previous studies have indicated that both anorexia nervosa (AN) and bulimia nervosa $(\mathrm{BN})$ are associated with

Received December 16, 2019; revised and accepted March 13, 2020. Published online March 27, 2020; doi: 10.1620/tjem.250.191.

Correspondence: Kyoko Kasahara, M.D., Ph.D., Department of Obstetrics and Gynecology, Shiga University of Medical Science, Seta

Tsukinowa-cho, Otsu, Shiga 520-2192, Japan.

e-mail: kasabee@belle.shiga-med.ac.jp

C 2020 Tohoku University Medical Press. This is an open-access article distributed under the terms of the Creative Commons Attribution-NonCommercial-NoDerivatives 4.0 International License (CC-BY-NC-ND 4.0). Anyone may download, reuse, copy, reprint, or distribute the article without modifications or adaptations for non-profit purposes if they cite the original authors and source properly.

https://creativecommons.org/licenses/by-nc-nd/4.0/ 
premature birth and small-for-gestational-age (SGA) infants, with the former subtype being more prominent (Linna et al. 2014). Maternal low weight is generally considered a predictor of these complications (Fujiwara et al. 2014; Enomoto et al. 2016; Nomura et al. 2017), not only in mothers with eating disorders (Linna et al. 2014). Psychological stress may be another factor that leads to pregnancy-related complications in mothers with eating disorders (Rondo et al. 2003; Linna et al. 2014).

However, the effects of maternal eating disorders on perinatal outcomes remain controversial. Recent studies with larger samples have failed to show an elevated risk of SGA or premature births in association with maternal eating disorders (Micali et al. 2012; O'Brien et al. 2017). Although smoking during pregnancy has been associated with SGA and premature births in the general population (Abraham et al. 2017; Tong et al. 2017), previous investigations on maternal eating disorders have suggested only a partial mediation effect of smoking on adverse fetal outcomes (Micali et al. 2016). Both remission and relapse have been reported among pregnant women with $\mathrm{AN}$, and remain controversial issues (Koubaa et al. 2005; Micali et al. 2007; Hoffman et al. 2011; Kimmel et al. 2016).

The aim of the present study was to assess perinatal outcomes and to explore methods to improve them in Japanese women with a history of AN. We focused on AN because we expected it to be both predictive of poorer perinatal outcomes compared with other types of eating disorders, and highly associated with the morbidity and mortality of infants (Kramer 1987; Barker et al. 1993; Baker et al. 2008; Longo et al. 2013; Katanoda et al. 2017). We hypothesized that Japanese women with a history of AN would give birth to smaller and more premature infants than unexposed women secondary to maternal low weight and smoking during pregnancy, which would be closely related.

\section{Methods}

The present study consists of a case series and a crosssectional study carried out using medical records in a single center. The confidentiality of the patients involved was protected. The local ethics committee of Shiga University of Medical Science Hospital approved the study (certificate approval numbers: R2018-070, R2019-187). We provided opt-out opportunities on the website of our institution for an adequate duration for any woman who had given birth but did not want to be included in the study.

\section{Study population}

The study population consisted of women who gave birth at Shiga University of Medical Science Hospital from April 1998 to March 2018. Over this 20-year period, 6,342 women gave birth from at least 22 weeks gestation at our hospital.

The case group was defined as Japanese women with a history of AN who had a singleton birth during the study period. First, we manually identified these women from hospital records by searching the diagnostic name of eating disorders or AN.

Second, we carefully identified the women who had been diagnosed with $\mathrm{AN}$, excluding those with other types of eating disorders or other psychiatric disorders. In addition, we read their records carefully and confirmed their diagnosis of AN in accordance with the American Psychiatric Association's Diagnostic and Statistical Manual, fifth edition (DSM-5) (American Psychiatric Association 2013).

Third, we excluded foreign women and those with conditions related to premature birth or growth restriction. The exclusion criteria were foreign names, multiple pregnancies, induced premature birth due to complicated pregnancy (e.g., placenta previa, placental abruption), stillbirth, serious congenital disorders, and the following pregnancy complications: hypertensive disorders including preeclampsia, diabetes, systemic lupus erythematosus, anti-phospholipid antibody syndrome, hyperthyroidism, untreated hypothyroidism, inflammatory bowel disease, and uterine malformation. We did not exclude patients who had received treatment for hypothyroidism before and during pregnancy or those who had delivered infants with minor malformations (e.g., cleft lip or palate, hydrocele testis, ventricular septal defect not requiring surgery).

We aimed to define the control group as Japanese women who had singleton births without a history of eating disorders or any conditions related to premature birth or SGA in the study population. These women were selected from the delivery register of Shiga University of Medical Science Hospital from April 2008 to March 2018. We utilized electronic medical records for patients from 2010 onward. Unfortunately, some paper-based records from before 2010 were incomplete or missing. Thus, the women in the control group were mainly selected from the period when electronic medical records were used. After excluding those who met the exclusion criteria, we randomly selected 240 unexposed women from the delivery register as the control group. We selected the first and second delivery case of every month from the 10-year delivery register, skipping cases that met the exclusion criteria, including foreign women and those with conditions related to premature birth or SGA, as the cases were applied. We also excluded patients with a history of any type of eating disorder and cases with missing data.

\section{Outcomes and potential confounders}

Premature birth, and the standard deviations (SDs) from the mean birth weight and mean head circumference at birth were evaluated as outcome measures. In order to evaluate prematurity, we preferred to use "premature birth," which is a dichotomized variable defined as childbirth earlier than 37 weeks of gestation, rather than "gestational week at birth" which is a continuous variable, because the frequency of planned cesarean section at 37 or 38 weeks of gestation due to previous cesarean section or uterine sur- 
gery was considerable in both groups. The SDs from mean birth weight and mean head circumference at birth, both continuous variables, were selected as indices of infant size at birth.

To express maternal low weight before and during pregnancy, pre-pregnancy body mass index (BMI) and gestational weight gain were adopted as potential confounders in the statistical analysis because they are associated with premature birth and infant size in the general population (Naeye and Tafari 1983; Fujiwara et al. 2014; Enomoto et al. 2016; Lima et al. 2018). For the analysis of SDs from mean birth weight and mean head circumference at birth, both of which can have hereditary effects, maternal height was also adopted as a potential confounder (Naeye and Tafari 1983).

\section{Data extraction}

Data were collected from the medical records of each patient. Maternal height, pre-pregnancy weight, and weight immediately before delivery were obtained to calculate prepregnancy BMI and gestational weight gain. Smoking history and fertility treatment history, including ovulation induction, artificial insemination, and assisted reproductive technology, were also investigated. SDs from mean birth weight and mean head circumference at birth were determined after assigning gestational weeks and days at birth, parity, and gender to "New Japanese neonatal anthropometric charts" (Itabashi et al. 2010, 2014). The clinical histories of the case group were surveyed in detail. Regular menstruation was confirmed based on the "self-reported" regularity in the questionnaire, or physician's description, and became definite when the expected date of delivery (EDD), determined from fetal size in early pregnancy, corresponded with the EDD calculated by last menstrual period. Anorexia behaviors within the 6 months prior to pregnancy were interpreted as symptoms before pregnancy, and we evaluated whether each case deteriorated during pregnancy or not according to the medical record.

\section{Statistical analysis}

The baseline patient characteristics for each group were summarized by mean and SD for continuous variables and absolute and relative frequencies (\%) for categorical variables. The Student's $t$ test for continuous variables and Fisher's exact test for categorical variables were used to compare the two groups. The outcomes and potential confounders for the statistical analysis were similarly summarized.

To compare the two groups, the odds ratios (ORs) for premature birth and the differences between two means for the SDs from mean birth weight and mean head circumference at birth were estimated, with their $95 \%$ confidence intervals, by logistic regression models for premature birth and linear regression models for the SDs from mean birth weight and mean head circumference at birth. The $P$ values or the tests for the ORs or the difference between the two means were calculated using these models.

First, the crude OR for premature birth and the crude differences between the two means for the SDs from mean birth weight and mean head circumference at birth were estimated using univariate models. Second, the adjusted ORs or the adjusted differences between the two means for the above outcomes were estimated using two approaches, multivariate models and matching analysis, to investigate whether the above outcome was associated with maternal AN after adjustment. For both approaches, we adjusted for pre-pregnancy BMI and gestational weight gain for premature birth, and pre-pregnancy BMI, gestational weight gain and height for the SDs from the mean birth weight and mean head circumference at birth. For the matching analysis, four controls per case were selected by matching with confounders using adjustment.

Statistical analysis was performed using EZR software (version 1.37; Saitama Medical Center, Jichi Medical University, Japan) (Kanda 2013). A two-sided significance level of $5 \%$ was used for all tests. For the matching analysis, matched controls were also selected from 240 unexposed women using EZR.

\section{Results}

We identified 17 pregnancies in 14 women with a history of AN from 20 years of hospital records. Two women with twin births and one with type I diabetes mellitus who delivered two singleton babies were excluded. A woman receiving treatment for hypothyroidism and another who delivered an otherwise healthy infant with a cleft lip and palate were included. Finally, we identified 13 singleton pregnancies in 11 Japanese women with a history of AN as the case group.

A comparison of the baseline characteristics of the study group is shown in Table 1. No differences in age, parity, or height were observed between the two groups, whereas the cases showed significantly lower pre-pregnancy weight $(P<0.001)$ than the controls. Both groups had a comparable frequency of fertility treatment history. The smoking rates for the case group were significantly higher than those for the control group, both before and during pregnancy (both $P<0.001$ ). No significant differences were seen in the mode of delivery or gender of the infants between the two groups. Compared with the control group, gestational week at childbirth was significantly earlier $(P<0.03)$, and both birth weight and head circumference at birth were significantly smaller in the case group ( $P$ $<0.002$ and $P<0.03$, respectively).

A comparison of the outcomes and potential confounders of the statistical analysis between the two groups, first in the total population, and then in nonsmokers during pregnancy, is shown in Table 2. In the total population, the frequency of premature birth was significantly higher $(P<$ 0.03 ), and the SDs from mean birth weight and mean head circumference at birth were significantly smaller in the case group $(P<0.007$ and $P<0.05$, respectively). In nonsmok- 
Table 1. Baseline characteristics of the two study groups.

\begin{tabular}{lccc}
\hline & $\begin{array}{c}\text { Case group } \\
(n=13)\end{array}$ & $\begin{array}{c}\text { Control group } \\
(n=240)\end{array}$ & $P$ value \\
\hline Age (y) at childbirth, mean (SD) & $31.0(4.9)$ & $33.0(5.2)$ & 0.17 \\
Parity, mean (SD) & $0.38(0.65)$ & $0.64(0.84)$ & 0.28 \\
Height (cm), mean (SD) & $157.8(6.8)$ & $158.7(5.1)$ & 0.52 \\
Pre-pregnancy weight (kg), mean (SD) & $43.7(5.5)$ & $51.8(7.6)$ & $<0.001^{* * *}$ \\
Fertility treatment, $n$ (\%) & $3(23.1)$ & $53(22.1)$ & 1 \\
Smoking before pregnancy, $n(\%)$ & $7(53.8)$ & $28(11.7)$ & $<0.001^{* * *}$ \\
Smoking during pregnancy, $n(\%)$ & $4(30.8)$ & $2(0.8)$ & $<0.001^{* * *}$ \\
Cesarean delivery, $n$ (\%) & $3(23.1)$ & $76(31.7)$ & 0.76 \\
Male infant, $n$ (\%) & $9(69.2)$ & $126(52.5)$ & 0.27 \\
Gestational week at childbirth, mean (SD) & $37.2(2.2)$ & $38.3(1.6)$ & $<0.03^{*}$ \\
Infant size at birth & & & $<0.002^{* *}$ \\
Birth weight $(\mathrm{g})$, mean (SD) & $2,520.6(530.9)$ & $2,910.1(417.0)$ & $<0.03^{*}$ \\
Head circumference (cm), mean (SD) & $32.08(1.56)$ & $33.05(1.47)$ & \\
\hline
\end{tabular}

$\mathrm{SD}$, standard deviation.

${ }^{*} P<0.05,{ }^{* *} P<0.01,{ }^{* * *} P<0.001$.

Table 2. Comparison of outcomes and potential confounders in the case group and control group in (a) the total population and (b) nonsmokers during pregnancy.

\begin{tabular}{|c|c|c|c|}
\hline & Case group & Control group & $P$ value \\
\hline \multicolumn{4}{|l|}{ (a) Total populations } \\
\hline Number & 13 & 240 & \\
\hline \multicolumn{4}{|l|}{ Potential confounders } \\
\hline Pre-pregnancy BMI $\left(\mathrm{kg} / \mathrm{m}^{2}\right)$, mean (SD) & $17.49(1.64)$ & $20.58(3.01)$ & $<0.001^{* * *}$ \\
\hline Gestational weight gain $(\mathrm{kg})$, mean $(\mathrm{SD})$ & $2.72(5.27)$ & $9.84(5.69)$ & $<0.001^{* * *}$ \\
\hline \multicolumn{4}{|l|}{ Outcomes } \\
\hline Premature birth, $n(\%)$ & $4(30.8)$ & $20(8.3)$ & $<0.03^{*}$ \\
\hline Infant size at birth & $17.49(1.64)$ & $20.58(3.01)$ & $<0.001^{* * *}$ \\
\hline SD from mean birth weight, mean (SD) & $-0.72(1.22)$ & $0.04(0.96)$ & $<0.007^{* *}$ \\
\hline SD from mean head circumference, mean (SD) & $-0.56(0.72)$ & $0.00(1.02)$ & $<0.05^{*}$ \\
\hline \multicolumn{4}{|l|}{ (b) Nonsmokers during pregnancy } \\
\hline Number & 9 & 238 & \\
\hline \multicolumn{4}{|l|}{ Potential confounders } \\
\hline Pre-pregnancy BMI $\left(\mathrm{kg} / \mathrm{m}^{2}\right)$, mean (SD) & $17.58(1.48)$ & $20.60(3.02)$ & $<0.004^{* *}$ \\
\hline Gestational weight gain $(\mathrm{kg})$, mean $(\mathrm{SD})$ & $2.66(3.63)$ & $9.76(5.63)$ & $<0.001^{* * *}$ \\
\hline \multicolumn{4}{|l|}{ Outcomes } \\
\hline Premature birth, $n(\%)$ & $3(33.3)$ & $20(8.4)$ & $<0.05^{*}$ \\
\hline \multicolumn{4}{|l|}{ Infant size at birth } \\
\hline SD from mean birth weight, mean (SD) & $-0.60(1.06)$ & $0.04(0.96)$ & 0.05 \\
\hline SD from mean head circumference, mean (SD) & $-0.50(0.77)$ & $0.00(1.02)$ & 0.15 \\
\hline
\end{tabular}

BMI, body mass index; SD, standard deviation.

${ }^{*} P<0.05,{ }^{* *} P<0.01,{ }^{* * *} P<0.001$.

ers, the frequency of premature birth was significantly higher in the case group $(P<0.05)$, but the cases did not show a significantly smaller SD from mean birth weight or mean head circumference at birth compared to the controls ( $P=0.05$ and $P=0.15$, respectively). On the other hand, pre-pregnancy BMI and gestational weight gain were significantly smaller in the case group in nonsmokers $(P<$ 0.004 and $P<0.001$, respectively), as well as in the total population (both $P<0.001$ ).

The detailed profiles of the case group are shown in 
Table 3. Detailed profiles of the case group.

\begin{tabular}{|c|c|c|c|c|c|c|c|c|c|c|c|c|c|}
\hline No. & 1 & 2 & 3 & 4 & 5 & 6 & 7 & 8 & 9 & 10 & 11 & 12 & 13 \\
\hline Age (y) at childbirth & 23 & 28 & 28 & 37 & 31 & 36 & 36 & 35 & 36 & 27 & 34 & 28 & 24 \\
\hline Parity & 2 & 0 & 0 & 1 & 1 & 0 & 0 & 1 & 0 & 0 & 0 & 0 & 0 \\
\hline Mode of delivery & VD & VD & VD & $\mathrm{CS}$ & $\mathrm{CS}$ & VD & $\mathrm{CS}$ & VD & VD & VD & VD & VD & VD \\
\hline Changing hospital & + & - & - & - & + & - & - & - & + & + & + & + & - \\
\hline Minimum BMI in the past $\left(\mathrm{kg} / \mathrm{m}^{2}\right)$ & NA & 15.2 & 14.5 & 14.6 & NA & NA & 14.6 & NA & 13.9 & 16.3 & NA & 16.8 & 14.5 \\
\hline Age $(y)$ at minimum BMI & & 20 & 25 & 32 & & & 32 & & 18 & 26 & & 16 & 20 \\
\hline Pre-pregnancy BMI (kg/m²) & 16.8 & 16.3 & 19.9 & 15.5 & 16.4 & 16.7 & 16.4 & 17.1 & 16.75 & 19.0 & 16.4 & 19.6 & 20.5 \\
\hline Gestational weight gain $(\mathrm{kg})$ & -4.8 & 5.6 & 4.25 & 3.85 & -2.3 & -4.9 & 1.6 & 0.85 & 3.7 & 5.4 & 0.4 & 7 & 14.75 \\
\hline Regular menstruation & + & - & + & - & - & - & - & - & - & - & - & - & + \\
\hline Fertility treatment & - & - & - & - & + & - & - & - & + & - & + & - & - \\
\hline Smoking before pregnancy & + & + & - & + & + & - & + & - & - & - & + & - & + \\
\hline Smoking during pregnancy & + & - & - & + & + & - & - & - & - & - & - & - & + \\
\hline Symptoms before pregnancy & + & - & - & + & + & - & + & - & + & + & - & - & + \\
\hline Worsening during pregnancy & + & + & + & + & + & + & - & + & - & - & + & - & + \\
\hline \multicolumn{14}{|l|}{ Symptoms during pregnancy } \\
\hline Hyperemesis gravidarum & - & - & + & - & NA & + & - & - & NA & NA & - & + & - \\
\hline \multicolumn{14}{|l|}{ Anorexia symptoms } \\
\hline Binge eating & + & - & - & + & - & - & + & - & + & - & - & - & + \\
\hline Purging behavior & + & - & + & + & + & - & + & - & + & - & - & - & + \\
\hline Restrictive eating & - & + & + & - & + & + & - & + & - & + & + & - & - \\
\hline Gestational week at childbirth & 37 & 40 & 40 & 37 & 33 & 39 & 35 & 34 & 37 & 38 & 36 & 39 & 39 \\
\hline \multicolumn{14}{|l|}{ Infant size at birth } \\
\hline SD from mean birth weight & -2.2 & -2.1 & -1.6 & -1.6 & -1.6 & -1.1 & -0.9 & -0.8 & -0.7 & -0.2 & 0.8 & 1.2 & 1.5 \\
\hline SD from mean head circumference & -1.6 & -1.5 & -1.1 & -0.7 & -0.6 & 0.5 & 0 & -1.1 & -1.2 & -0.4 & -0.3 & 0.6 & 0.1 \\
\hline
\end{tabular}

BMI, body mass index; CS, cesarean section; NA, not available; SD, standard deviation; VD, vaginal delivery.

Table 3. The cases are listed in order of decreasing SD from mean birth weight; No. 4 and No. 7, and No. 8 and No. 11 were the same women. Three cases underwent planned cesarean section for a breech baby (No. 7) or previous cesarean section (No. 4 and No. 5). No case suffered non-reassuring fetal status or low Apgar scores $(\leq 7)$ in their newborn.

Those who changed hospital during pregnancy due to anorexia symptoms tended to lack information on their minimum BMI in the past. Nine cases became pregnant with a BMI of less than $18.5 \mathrm{~kg} / \mathrm{m}^{2}$, which corresponds to the World Health Organization's threshold for "underweight" (World Health Organization 2006). Three women in the case group (No. 1, No. 3, and No. 13) had regular menstruation, and only two (No. 3 and No. 13) had both regular menstruation and normal weight before pregnancy. Among the nine underweight cases, six women became pregnant spontaneously; though five of them did not have regular menstruation (No. 2, No. 4, No. 6, No. 7, and No. 8). Three underweight cases without regular menstruation (No. 5, No. 9, and No. 11) conceived owing to fertility treatment and one, No. 9, underwent in vitro fertilization.

Seven cases had anorexia symptoms before pregnancy. Nine cases got worse during pregnancy, and all cases other than No. 12 presented with anorexia symptoms during preg- nancy. Five cases (No. 1, No. 4, No. 7, No. 9, and No. 13) seemed to be binge-purge type, and seven (No. 2, No. 3, No. 5, No. 6, No. 8, No. 10, and No. 11) were thought to be restricting type. The subtypes of $\mathrm{AN}$ had no relation to prepregnancy BMI, gestational weight gain, prematurity, or infant size (data not shown).

Among the seven smokers, three stopped smoking after they became pregnant. The four women who continued to smoke during pregnancy (No. 1, No. 4, No. 5, and No. 13) had three points in common: anorexia symptoms before pregnancy, deterioration during pregnancy, and purging behavior. Three (No. 1, No. 4, and No. 5) became pregnant while underweight and delivered SGA infants still being underweight. No. 1 and No. 5 lost weight before childbirth, and No. 4 had the lowest pre-pregnancy BMI among those in the case group. On the other hand, No. 13 became pregnant with normal weight, and delivered a baby with a birth weight of 1.5 SD from the mean, having gained much weight during pregnancy, probably mediated by binge eating quantitatively more than purging behavior. Thus, smoking during pregnancy was found to be involved with extremely disturbed eating behaviors, though not always related to low BMI and less weight gain.

Next, we evaluated premature birth and infant size at birth in maternal AN including pregnant smokers, because 
excluding smokers would leave only mild cases of maternal AN.

\section{Premature birth}

Maternal AN was positively associated with the frequency of premature birth $(\mathrm{OR}=4.89, P<0.02)$. However, this positive association was not observed after adjustment for pre-pregnancy BMI and gestational weight gain $(\mathrm{OR}=$ $0.202, P=0.11$ ), or in the matching analysis with pre-pregnancy $\mathrm{BMI}$ and gestational weight $(\mathrm{OR}=1.87, P=0.37)$ (see Table 4).

\section{Infant size at birth}

The difference in means of SDs from mean birth weight between the two groups was statistically significant (mean difference $[\mathrm{MD}]=0.758, P<0.007$ ), whereas adjustment for pre-pregnancy BMI, gestational weight gain, and height reduced the MD, and the adjusted MD between the two groups was not statistically significant $(\mathrm{MD}=0.176, P$ $=0.52$ ). Similarly, the matching analysis with pre-pregnancy BMI, gestational weight gain, and height revealed a smaller MD than the crude MD, and no significant difference was observed between the two groups $(\mathrm{MD}=0.385, P$ $=0.20)$ (see Table 5).

Analysis of the SDs from mean head circumference at birth revealed a statistically significant difference between the means in the case compared to the control group ( $\mathrm{MD}=$ $0.564, P<0.05$ ), whereas adjustment for pre-pregnancy BMI, gestational weight gain, and height, as well as the matching analysis with these three confounders, reduced the difference between the two means and revealed no significant difference between the two groups ( $\mathrm{MD}=0.307, P$ $=0.31 ; \mathrm{MD}=0.200, P=0.56)($ see Table 6$)$.

Table 4. Premature births in maternal anorexia nervosa compared to controls: crude, adjusted, and matched odds ratios (ORs) and $95 \%$ confidence intervals (CIs) calculated by logistic regression.

\begin{tabular}{lcccc}
\hline & OR & $95 \% \mathrm{CI}$ & $P$ value & No. of controls \\
\hline Crude & 4.89 & $1.38-17.3$ & $<0.02^{*}$ & 240 \\
Adjusted $^{\dagger}$ & 0.202 & $0.0286-1.42$ & 0.11 & 240 \\
Matched $^{\ddagger}$ & 1.87 & $0.477-7.31$ & 0.37 & 52 \\
\hline
\end{tabular}

BMI, body mass index.

${ }^{\uparrow}$ Adjusted for pre-pregnancy BMI and gestational weight gain. ${ }^{\star}$ Matched on pre-pregnancy BMI and gestational weight gain. ${ }^{*} P<0.05$

Table 5. Standard deviation from mean birth weight in maternal anorexia nervosa compared to controls: crude, adjusted, and matched mean differences (MDs) and 95\% confidence intervals (CIs) calculated by linear regression.

\begin{tabular}{lcccc}
\hline & MD & $95 \%$ CI & $P$ value & No. of controls \\
\hline Crude & 0.758 & $0.213-1.303$ & $<0.007^{* *}$ & 240 \\
Adjusted $^{\dagger}$ & 0.176 & $-0.364-0.716$ & 0.52 & 240 \\
Matched $^{\ddagger}$ & 0.385 & $-0.208-0.977$ & 0.20 & 52 \\
\hline
\end{tabular}

BMI, body mass index.

${ }^{\dagger}$ Adjusted for pre-pregnancy BMI, gestational weight gain, and height.

¥Matched on pre-pregnancy BMI, gestational weight gain, and height.

${ }^{* *} P<0.01$.

Table 6. Standard deviation from mean head circumference at birth in maternal anorexia nervosa compared to controls: crude, adjusted, and matched mean differences (MDs) and 95\% confidence intervals (CIs) calculated by linear regression.

\begin{tabular}{lcccc}
\hline & MD & $95 \%$ CI & $P$ value & No. of controls \\
\hline Crude & 0.564 & $0.001-1.126$ & $<0.05^{*}$ & 240 \\
Adjusted $^{\dagger}$ & 0.307 & $-0.288-0.902$ & 0.31 & 240 \\
Matched $^{\ddagger}$ & 0.200 & $-0.476-0.816$ & 0.56 & 52 \\
\hline
\end{tabular}

BMI, body mass index.

${ }^{\dagger}$ Adjusted for pre-pregnancy BMI, gestational weight gain, and height.

${ }^{\star}$ Matched on pre-pregnancy BMI, gestational weight gain, and height.

${ }^{*} P<0.05$. 


\section{Discussion}

This study has demonstrated, in a Japanese sample, that anorexia symptoms during pregnancy were quite common in women with a history of AN, and that maternal AN was associated with an increased risk of both premature birth and symmetric growth restriction. We also confirmed statistically that these adverse effects were mediated by maternal low pre-pregnancy BMI and small gestational weight gain. Smoking during pregnancy was more common in the cases than in the controls and could have an additive effect on adverse perinatal outcomes resulted from severe eating disorder.

A major limitation of our study was the small sample size. A large-scale study of maternal eating disorders is difficult to carry out in Japan. About 1 million neonates are born in Japan every year, nearly half of whom are delivered in small-scale private clinics (Mother's and Children's Health and Welfare Association 2019), representing a delay in consolidation of delivery facilities. This makes a largescale, single-center study of a complicated pregnancy with low frequency difficult to perform in Japan. Moreover, the Japan Perinatal Registry Network database, a public database, does not include a history of eating disorders as a survey item. Therefore, we used statistical techniques, after setting up an adequate number of controls, to overcome the limitations of this small-scale study.

First, comparing the case and control groups, we confirmed that maternal AN in Japan was associated with an increased risk of premature birth and symmetric growth restriction (Tables 1 and 2). Second, we aimed to identify the factors responsible for adverse effects, independent of the characteristics of the study population, which have often caused controversy in observational studies (Micali et al. 2012; O'Brien et al. 2017). We adopted two statistical approaches: multivariate models adjusted for the confounders indicating maternal low weight before and during pregnancy, and a matching analysis by the selection of matched controls with the same confounders (Tables 4, 5 and 6). Because no positive association was observed between maternal AN with a high frequency of premature birth and small infant size after taking each approach, we concluded that these poor perinatal outcomes were affected by maternal low weight before and during pregnancy.

We also examined the profiles of the case group closely by making the best use of a small-scale study (Table 3). The case group in the present study showed a high prevalence of anorexia symptoms during pregnancy, with more than half of the cases experiencing worsening AN symptoms during pregnancy. In most cases, the anorexia symptoms were supposed to be associated with less gestational weight gain and even with weight loss before childbirth.

The smoking rates for the case group were higher than those for the control group both before and during pregnancy (Table 1), in accord with previous reports (Bulik et al. 2009). In the linear regression analysis of the SDs from mean birth weight and mean head circumference at birth, adjustment for smoking during pregnancy diminished the statistically significant difference between the two groups (data not shown). Among nonsmokers, those in the case group showed a lower pre-pregnancy BMI and smaller gestational weight gain, but not a smaller infant size (Table 2). At first glance, these results may indicate that smoking during pregnancy, rather than maternal low weight, would adversely affect perinatal outcomes. However, detailed profiles of the case group demonstrated the distinctive features of those who continued to smoke despite pregnancy. These patients conceived with chronic anorexia symptoms and deteriorated during pregnancy, presenting with purging behavior (Table 3). Among pregnant smokers, one woman crossed over from $\mathrm{AN}$ to $\mathrm{BN}$, leading to excessive weight gain and the birth of a large-for-date infant, while others presented extremely low weight and delivered SGA infants. We therefore presumed that smoking during pregnancy might be associated with extremely disturbed eating behaviors. Excluding pregnant smokers would leave mild cases of maternal AN without affecting the evaluation of prepregnancy BMI and gestational weight gain (Table 2). Thus, we included smokers when evaluating perinatal outcomes in maternal AN (Tables 4, 5 and 6).

As is well known, smoking itself has deleterious effects on the fetal growth (Abraham et al. 2017; Tong et al. 2017). Besides that, after considering the limitations due to the small-scale samples, we feel that smoking during pregnancy may be an indicator of abnormal eating behavior, and both could adversely affect perinatal outcomes. In other words, smoking would be a predictor of poor perinatal outcomes through both the severe anorexia symptoms of the smoking mothers, as well as a serious threat to the fetus via the well-known negative effects of nicotine and carbon monoxide. Thus, by close examination of small-scale samples, we exposed the relationship between smoking and perinatal outcomes in maternal AN. Our result may correspond with the previous multivariate analysis with large samples employing smoking as a covariate, and could explain their result, that is, a partial effect of smoking on the infant size at birth in maternal AN (Micali et al. 2016). Generally, smoking cessation in pregnancy is recommended for adequate weight gain and normal fetal growth (Abraham et al. 2017; Tong et al. 2017). On the other hand, our results indicated that interventions for smoking cessation, without considering latent problems involving eating behaviors, could easily fail. This is due to the fact that smoking could be not only a body image-related issue, but also a sign of the physical, psychological and social pathological severity of eating disorders (Levine et al. 2001, 2010; Fulkerson and French 2003). Therefore, we suggest that successful psychiatric treatment and the amelioration of anorexia symptoms before pregnancy could improve perinatal outcomes and decrease the risk of poor health in infants through both normal nutrition and smoking cessa- 
tion. Maternal smoking (Abraham et al. 2017; Tong et al. 2017), chronic malnutrition, and hypercortisolemia resulting from a stress reaction due to disturbed self-image during pregnancy, have already been reported to be major candidates for symmetric growth restriction in maternal AN (Lou et al. 1994; Koubaa et al. 2005; Micali et al. 2016). Small head circumference at birth, similarly to low birth weight may have a detrimental effect on brain development (Koubaa et al. 2005), and might be associated with an increased risk of coronary heart disease in adulthood (Risnes et al. 2009).

Of note, more than half of the cases became pregnant while "underweight" (World Health Organization 2006), lower than approximately $90 \%$ of standard body weight, which was previously reported as the average weight at which resumption of menstruation occurs in AN (Golden et al. 1997). In addition, our result showed that such underweight patients often conceived spontaneously without regular menstruation. These findings indicated not only that fertility could often resume unexpectedly in women with $\mathrm{AN}$, implying the risk of an unplanned pregnancy (Bulik et al. 2010), but also that thin women who were otherwise healthy could have a past history of eating disorders. As women with a history of eating disorders are often apparently healthy and tend to hide their past histories from maternity care providers (Fogarty et al. 2018), thin women should be observed carefully during perinatal visits so that potential disturbed eating behaviors are not overlooked. In our experience, weight loss in mid- or late pregnancy might predict anorexia symptoms (data not shown), while weight loss due to hyperemesis gravidarum is common in early pregnancy. A recent study reported that gestational weight gain had a greater effect than pre-pregnancy BMI on the increase in birth weight (Lima et al. 2018), which should encourage underweight pregnant women to gain adequate weight and deliver healthy babies.

Also to be considered is the issue of fertility treatment for underweight women with a history of AN. The advances in fertility treatment have brought benefits to many women and fertility treatments have become widespread, especially in developed countries. The guidelines for the treatment of eating disorders in Japan (Japan Society for Eating Disorders 2012) refers to some comorbidities of AN, but does not include amenorrhea and infertility. In the standard textbook of obstetrics and gynecology in Japan (Japan Society of Obstetrics and Gynecology 2016), ovulation induction for $\mathrm{AN}$ is recommended in cases with higher than $85 \%$ of standard body weight, which is equivalent to $18.7 \%$ of BMI. However, unfortunately, such a recommendation is not always observed in clinical practice as our results show. All healthcare providers, particularly fertility doctors, should recognize the risk of a pregnancy complicated with active AN. It is possible that the cases who became pregnant while underweight or without regular menstruation in our study may still have had anorexia symptoms whether they reported them to their doctors or not. Moreover, ovulation induction carries the risk of a multiple pregnancy which is also associated with premature birth and SGA as well as further obstetric complications. In this study, both twin mothers with a history of AN, who were excluded from the case group, had undergone fertility treatment and presented these severe complications (data not shown). We would therefore suggest that the guidelines for pregnancy in women with a history of AN include the recommendation that ovulation induction be limited in patients with lower than $85 \%$ standard body weight. The cooperative medical care of pregnant women with AN by obstetricians and psychiatrists should be also recommended in order to prevent deterioration during pregnancy.

Another limitation of this study is that our hospital is a tertiary hospital providing a specialized, highly technical level of health care. Our findings might therefore be biased because our case group consisted of particularly severe cases, including patients transferred to our hospital due to deterioration during pregnancy. In addition, some women in the control group had minor physical or psychological problems that could be indirectly related to premature birth or low birth weight. The prevalence of fertility treatment in the control group would also be larger than in the general population.

In conclusion, anorexia symptoms during pregnancy were found to be associated with an increased risk of premature birth and symmetric growth restriction mediated by maternal low pre-pregnancy BMI and small gestational weight gain in Japanese women with a history of AN. It is conceivable that smoking during pregnancy is a potential indicator of the severity of eating behavior and is predictive of poor perinatal outcomes. Remission of anorexia symptoms, not only weight gain, but also psychological recovery before pregnancy, could improve perinatal outcomes through both normal nutrition and smoking cessation. Fertility treatment for the women with a history of AN while underweight is not recommended.

To our knowledge, the present study is the first to investigate perinatal outcomes in maternal AN in Japan. Because the consolidation of delivery facilities is not really advancing in our country, there is a limitation in conducting a large-scale study of a complicated pregnancy with low frequency in a single institution. Multi-institutional joint research or the Japanese Nurses' Health Study, an ongoing prospective cohort study involving female nurses (Katanoda et al. 2017), might reveal more meaningful outcomes regarding these issues based on large-scale samples in the future.

\section{Acknowledgments}

The authors sincerely thank the staff of the Center for Clinical Research and Advanced Medicine, Shiga University of Medical Science Hospital, for their contributions to the present study, particularly for their able assistance in statistical analysis. This research received no specific grant from any funding agency in the public, commercial, 
or not-for-profit sectors.

\section{Conflict of Interest}

The authors declare no conflict of interest.

\section{References}

Abraham, M., Alramadhan, S., Iniguez, C., Duijts, L., Jaddoe, V.W., Den Dekker, H.T., Crozier, S., Godfrey, K.M., Hindmarsh, P., Vik, T., Jacobsen, G.W., Hanke, W., Sobala, W., Devereux, G. \& Turner, S. (2017) A systematic review of maternal smoking during pregnancy and fetal measurements with meta-analysis. PLoS One, 12, e0170946.

American Psychiatric Association (2013) Diagnostic and Statistical Manual of Mental Disorders, 5th ed., American Psychiatric Association, Washington, DC.

Baker, J.L., Olsen, L.W. \& Sorensen, T.I. (2008) Weight at birth and all-cause mortality in adulthood. Epidemiology, 19, 197-203.

Barker, D.J., Gluckman, P.D., Godfrey, K.M., Harding, J.E., Owens, J.A. \& Robinson, J.S. (1993) Fetal nutrition and cardiovascular disease in adult life. Lancet, 341, 938-941.

Bulik, C.M., Hoffman, E.R., Von Holle, A., Torgersen, L., Stoltenberg, C. \& Reichborn-Kjennerud, T. (2010) Unplanned pregnancy in women with anorexia nervosa. Obstet. Gynecol., 116, 1136-1140.

Bulik, C.M., Von Holle, A., Siega-Riz, A.M., Torgersen, L., Lie, K.K., Hamer, R.M., Berg, C.K., Sullivan, P. \& ReichbornKjennerud, T. (2009) Birth outcomes in women with eating disorders in the Norwegian mother and child cohort study (MoBa). Int. J. Eat. Disord., 42, 9-18.

Enomoto, K., Aoki, S., Toma, R., Fujiwara, K., Sakamaki, K. \& Hirahara, F. (2016) Pregnancy outcomes based on pre-pregnancy body mass index in Japanese women. PLoS One, 11, e 0157081 .

Fogarty, S., Elmir, R., Hay, P. \& Schmied, V. (2018) The experience of women with an eating disorder in the perinatal period: a meta-ethnographic study. BMC Pregnancy Childbirth, 18, 121.

Fujiwara, K., Aoki, S., Kurasawa, K., Okuda, M., Takahashi, T. \& Hirahara, F. (2014) Associations of maternal pre-pregnancy underweight with small-for-gestational-age and spontaneous preterm birth, and optimal gestational weight gain in Japanese women. J. Obstet. Gynaecol. Res., 40, 988-994.

Fulkerson, J.A. \& French, S.A. (2003) Cigarette smoking for weight loss or control among adolescents: gender and racial/ ethnic differences. J. Adolesc. Health, 32, 306-313.

Golden, N.H., Jacobson, M.S., Schebendach, J., Solanto, M.V., Hertz, S.M. \& Shenker, I.R. (1997) Resumption of menses in anorexia nervosa. Arch. Pediatr. Adolesc. Med., 151, 16-21.

Hoffman, E.R., Zerwas, S.C. \& Bulik, C.M. (2011) Reproductive issues in anorexia nervosa. Expert Rev. Obstet. Gynecol., 6, 403-414.

Itabashi, K., Fujimura, M., Kusuda, S., Tamura, M., Hayashi, T., Takahashi, T., Goishi, K., Futamura, M., Takahashi, Y., Isobe, K., Iida, K., Uetani, Y., Kondo, Y., Shirahata, S., Sugiura, M., et al. (2010) Introduction of new Japanese reference curves for neonatal anthropometry by gestational age. J. Jpn. Pediatr. Soc., 114, 1271-1293 (in Japanese).

Itabashi, K., Miura, F., Uehara, R. \&Nakamura, Y. (2014) New Japanese neonatal anthropometric charts for gestational age at birth. Pediatr. Int., 56, 702-708.

Japan Society for Eating Disorders (2012) Practice Guideline for Eating Disorders. Japan Society for Eating Disorders, Tokyo (in Japanese).

Japan Society of Obstetrics and Gynecology (2016) The knowledge required for residents of obstetrics and gynecology 20162018. Japan Society of Obstetrics and Gynecology, Tokyo (in
Japanese).

Kanda, Y. (2013) Investigation of the freely available easy-to-use software 'EZR' for medical statistics. Bone Marrow Transplant., 48, 452-458.

Katanoda, K., Noda, M., Goto, A., Mizunuma, H., Lee, J.S. \& Hayashi, K. (2017) Impact of birth weight on adult-onset diabetes mellitus in relation to current body mass index: the Japan nurses' health study. J. Epidemiol., 27, 428-434.

Kimmel, M.C., Ferguson, E.H., Zerwas, S., Bulik, C.M. \& Meltzer-Brody, S. (2016) Obstetric and gynecologic problems associated with eating disorders. Int. J. Eat. Disord., 49, 260-275.

Koubaa, S., Hallstrom, T., Lindholm, C. \& Hirschberg, A.L. (2005) Pregnancy and neonatal outcomes in women with eating disorders. Obstet. Gynecol., 105, 255-260.

Kramer, M.S. (1987) Determinants of low birth weight: methodological assessment and meta-analysis. Bull. World Health Organ., 65, 663-737.

Levine, M.D., Marcus, M.D., Kalarchian, M.A., Houck, P.R. \& Cheng, Y. (2010) Weight concerns, mood, and postpartum smoking relapse. Am. J. Prev. Med., 39, 345-351.

Levine, M.D., Perkins, K.A. \& Marcus, M.D. (2001) The characteristics of women smokers concerned about postcessation weight gain. Addict. Behav., 26, 749-756.

Lima, R., Batista, R.F.L., Ribeiro, M.R.C., Ribeiro, C.C.C., Simoes, V.M.F., Lima Neto, P.M., Silva, A. \& Bettiol, H. (2018) Prepregnancy body mass index, gestational weight gain, and birth weight in the BRISA cohort. Rev. Saude Publica, 52, 46.

Linna, M.S., Raevuori, A., Haukka, J., Suvisaari, J.M., Suokas, J.T. \& Gissler, M. (2014) Pregnancy, obstetric, and perinatal health outcomes in eating disorders. Am. J. Obstet. Gynecol., 211, 392. e1-8.

Longo, S., Bollani, L., Decembrino, L., Di Comite, A., Angelini, M. \& Stronati, M. (2013) Short-term and long-term sequelae in intrauterine growth retardation (IUGR). J. Matern. Fetal Neonatal Med., 26, 222-225.

Lou, H.C., Hansen, D., Nordentoft, M., Pryds, O., Jensen, F., Nim, J. \& Hemmingsen, R. (1994) Prenatal stressors of human life affect fetal brain development. Dev. Med. Child Neurol., 36, 826-832.

Micali, N., De Stavola, B., dos-Santos-Silva, I., Steenweg-de Graaff, J., Jansen, P.W., Jaddoe, V.W., Hofman, A., Verhulst, F.C., Steegers, E.A. \& Tiemeier, H. (2012) Perinatal outcomes and gestational weight gain in women with eating disorders: a population-based cohort study. BJOG, 119, 1493-1502.

Micali, N., Stemann Larsen, P., Strandberg-Larsen, K. \& Nybo Andersen, A.M. (2016) Size at birth and preterm birth in women with lifetime eating disorders: a prospective population-based study. BJOG, 123, 1301-1310.

Micali, N., Treasure, J. \& Simonoff, E. (2007) Eating disorders symptoms in pregnancy: a longitudinal study of women with recent and past eating disorders and obesity. J. Psychosom. Res., 63, 297-303.

Mother's \& Children's Health \& Welfare Association (2019) Maternal and child health statistics of Japan, 2018. Boshihokenjigyōdan, Tokyo, Japan (in Japanese).

Naeye, R.L. \& Tafari, N. (1983) Risk factors in pregnancy and diseases of the fetus and newborn. Williams and Wilkins, Baltimore, MD.

Nakai, Y., Nin, K. \& Noma, S. (2014) Eating disorder symptoms among Japanese female students in 1982, 1992 and 2002. Psychiatry Res., 219, 151-156.

Nakai, Y., Nin, K., Noma, S., Teramukai, S., Fujikawa, K. \& Wonderlich, S.A. (2018) Changing profile of eating disorders between 1963 and 2004 in a Japanese sample. Int. J. Eat. Disord., 51, 953-958.

Nomura, K., Kido, M., Tanabe, A., Nagashima, K., Takenoshita, S. \& Ando, K. (2017) Investigation of optimal weight gain 
during pregnancy for Japanese women. Sci. Rep., 7, 2569.

O'Brien, K.M., Whelan, D.R., Sandler, D.P., Hall, J.E. \& Weinberg, C.R. (2017) Predictors and long-term health outcomes of eating disorders. PLoS One, 12, e0181104.

Risnes, K.R., Nilsen, T.I., Romundstad, P.R. \& Vatten, L.J. (2009) Head size at birth and long-term mortality from coronary heart disease. Int. J. Epidemiol., 38, 955-962.

Rondo, P.H., Ferreira, R.F., Nogueira, F., Ribeiro, M.C., Lobert, H. \& Artes, R. (2003) Maternal psychological stress and distress as predictors of low birth weight, prematurity and intrauterine growth retardation. Eur. J. Clin. Nutr., 57, 266-272.

Tong, V.T., England, L.J., Rockhill, K.M. \& D’Angelo, D.V. (2017) Risks of preterm delivery and small for gestational age infants: effects of nondaily and low-intensity daily smoking during pregnancy. Paediatr. Perinat. Epidemiol., 31, 144-148.

World Health Organization (2006) Global standard of body mass index.

http://apps.who.int/bmi/index.jsp?introPage=intro_3.html [Accessed: January 20, 2020]. 